\title{
DISPOSITIF EXPÉRIMENTAL EN ULTRA-VIDE PERMETTANT LE DÉPOT DE GOUGHES MINGES ET L'ÉTUDE « IN SITU » DE LEURS PROPRIÉTÉS GALVANOMAGNÉTIQUES
}

\author{
Par JEAN SALARDENNE, \\ Faculté des Sciences de Bordeaux. \\ (Reçu le 9 octobre 1938.)
}

\begin{abstract}
Résumé. - Le dispositif réalisé permet de préparer des couches minces par évaporation sous ultra-vide $\left(5 \times 10^{-10}\right.$ torr $)$, puis d'étudier, dans la même enceinte, les propriétés galvanomagnétiques de ces couches constamment maintenues en ultra-vide.

La température $\theta$ du support peut être contrôlée pendant le dépôt et pendant les mesures ; le support, en mica, peut être clivé sous ultra-vide, tandis qu'un creuset de forme spéciale permet un dégazage intensif avant l'évaporation; enfin, une régulation de la vitesse d'évaporation et une mesure des pressions partielles à l'aide d'un analyseur de gaz résiduels permettent le contrôle de la plupart des paramètres importants intervenant lors de la formation des couches.

Abstract. - The device constructed permits one to prepare thin films by evaporation in ultra high vacuum $\left(5 \times 10^{-13}\right.$ torr $)$, then, to measure the galvanomagnetic coefficients of these films, constantly held in ultra high vacuum.

The substrate temperature can be controlled during the evaporation; the mica-substrate may be cleaved in the ultra high vacuum while a specially shaped crucible permits an intensive outgassing before the evaporation; finally, regulation of the evaporation rate and the measurement of the partial pressures with a residual gas analyser, permits the control of most of the important parameters which have an influence on the formation of the films.
\end{abstract}

I. Introduction. - Des études antérieures sur les couches minces de bismuth et de tellure [1] ont montré, d'une part, l'influence de la pression résiduelle pendant la condensation et, d'autre part, celle, probable, de l'oxygène sur leurs propriétés galvanomagnétiques.

Nous avons, de ce fait, été amené à concevoir et réaliser un dispositif expérimental en ultra-vide, permettant le dépôt de couches minces et la mesure des coefficients galvanomagnétiques de ces couches, constamment maintenues sous le même vide.

Par ailleurs, la nécessité d'effectuer la condensation sur un support dont la surface soit aussi propre que possible nous a conduit à étudier un dispositif de clivage sous ultra-vide.

Enfin, le contrôle de la température du support aussi bien pendant la condensation (recherche des conditions épitaxiales) que pendant les mesures (étude des propriétés à différentes températures) nous a paru indispensable.

II. Description du dispositif expérimental. - 1. DisPOSITIF A ULTRA-VIDE. - Le groupe de pompage est un ensemble classique : pompe à sorption-pompe ionique $(80 \mathrm{l} / \mathrm{s})$-sublimateur de titane. Il permet d'atteindre, après étuvage, un vide limite inférieur à $5 \times 10^{-10}$ torr dans une enceinte en acier inox, cylindrique, de $300 \mathrm{~mm}$ de diamètre, dont la partie utile a une hauteur de $215 \mathrm{~mm}$. Cette enceinte, en plus des accessoires tels que passages rotatifs, hublot, etc., possède à sa partie inférieure un passage verre-métal permettant l'adaptation d'un tube de pyrex. La partie inférieure de ce tube de pyrex peut, grâce au mouvement vertical de translation commandé par un chariot élévateur sur le plateau duquel est fixée l'enceinte, être descendue dans un dewar contenant de l'azote liquide ( $f g .1$ et 2 ). Il est alors possible, en introduisant un gaz inerte dans l'enceinte, d'effectuer les mesures des coefficients galvanomagnétiques à des températures allant jusqu'à $-195^{\circ}$ C.

2. Support. Positionnement du support. - Le support est fixé à un bloc de cuivre, parallélépipède rectangle de dimensions $70 \times 26 \times 8 \mathrm{~mm}$, qui contient une résistance chauffante en tungstène et dont la température est mesurée à l'aide d'une sonde au platine noyée dans le cuivre. On peut ainsi faire varier la température de la couche entre l'ambiante et $+250^{\circ} \mathrm{C}$.

Un mouvement de translation verticale est com- 


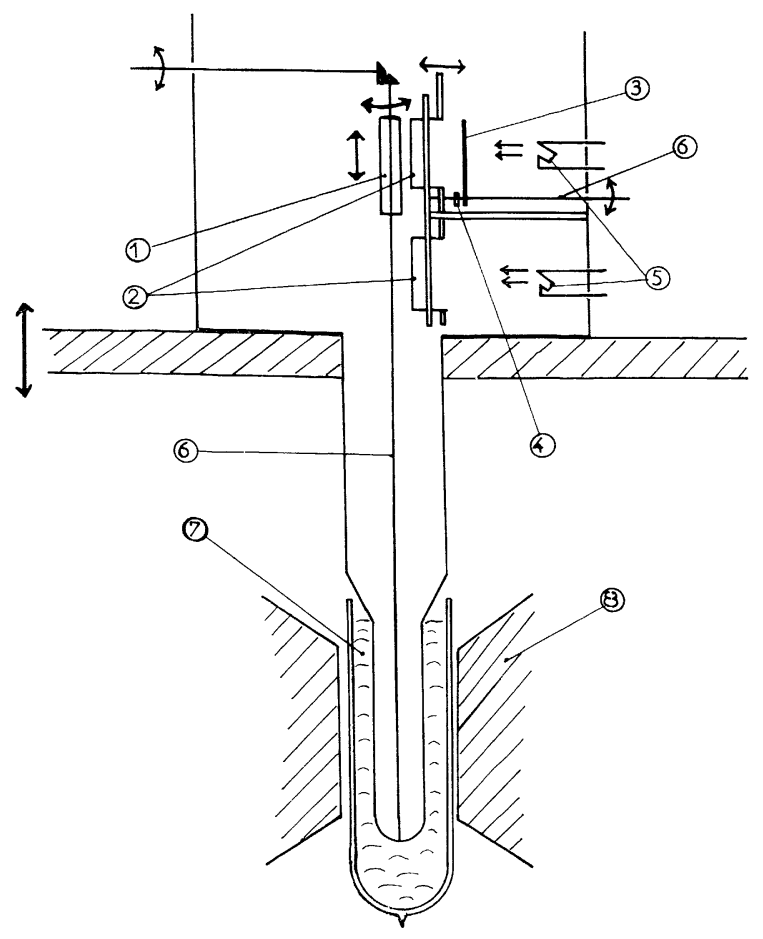

FIG. 1. - Schéma de principe : 1, Support ; 2, Caches ; 3 , Volet mobile; 4 , Butée provoquant l'ouverture du volet mobile en fin de course; 5 , Creusets; 6 , Vis sans fin; 7, Azote liquide; 8 , Électro-aimant.

mandé par la rotation d'une vis sans fin en acier « inox ». Il permet d'amener le support successivement aux positions I, II et III correspondant respectivement au dépôt de la couche mince, puis des électrodes et enfin à la mesure des coefficients galvanomagnétiques (fig. 1 et 2 ).

Lorsque le support est en position I ou II, un mouvement horizontal de translation des caches (destinés à donner à la couche et aux électrodes la forme voulue) permet de les amener au contact du support (afin que les contours soient bien nets). Ce même dispositif commande, en fin de course, l'ouverture ou la fermeture d'un volet mobile.

3. Dispositif de Glivage. - Ce dispositif, permettant le clivage des supports de mica juste avant l'évaporation, met à profit le mouvement vertical du support et consiste en un fil fin tendu, engagé dans le plan de clivage lors de l'installation dans l'enceinte.

4. Contacts aveg les fils de mesure. - Les contacts entre fils de mesure et couche mince sont réalisés par pression entre deux lamelles de mica dont l'une porte, en regard des électrodes de la couche mince, des revêtements à la laque d'argent reliés aux fils de mesure [1].

5. Creuset. - Le creuset contenant le matériau de la couche mince est normalement fermé et permet

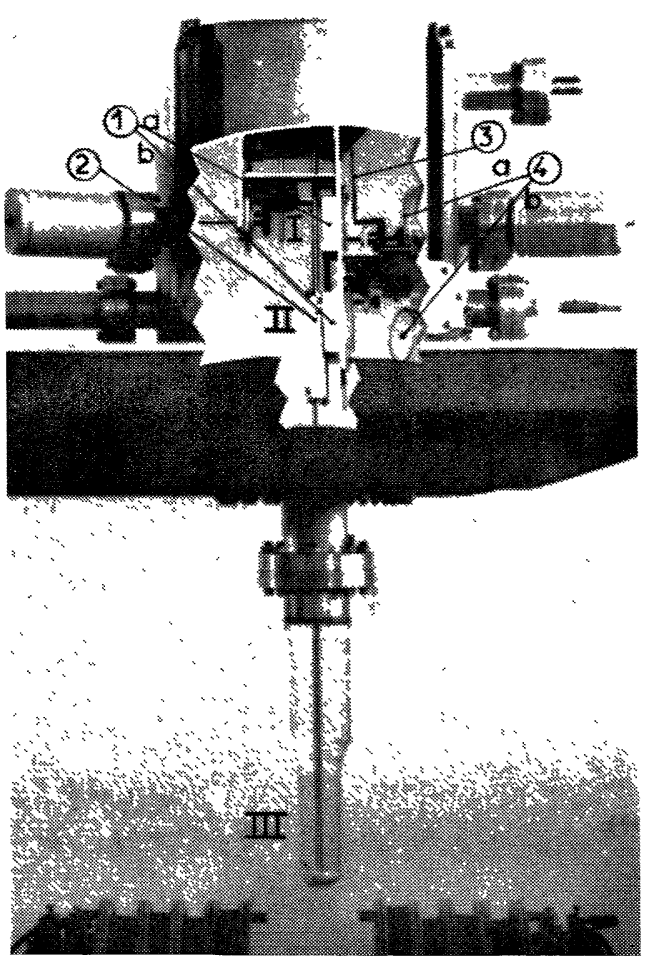

FIG. 2. - Réalisation : 1, Caches donnant leur forme : a) à la couche mince, b) aux électrodes ; 2 , Porte support ; 3, Volet mobile ; 4, Orifices d'évaporation : a) couche mince, b) électrodes.

Support en position I : dépôt de la couche mince. Support en position II : dépôt des électrodes.

Support en position III : mesures (le tube de pyrex étant plongé dans l'entrefer de l'électro-aimant).

ainsi, dans un premier temps, un dégazage intensif, par évaporation sans perte, du matériau à déposer. Lors de la réalisation de la couche mince, une commande d'ouverture du creuset est effectuée, thermiquement, par la dilatation amplifiée de la bande de tantale qui assurait primitivement l'obturation ( fig. 3).

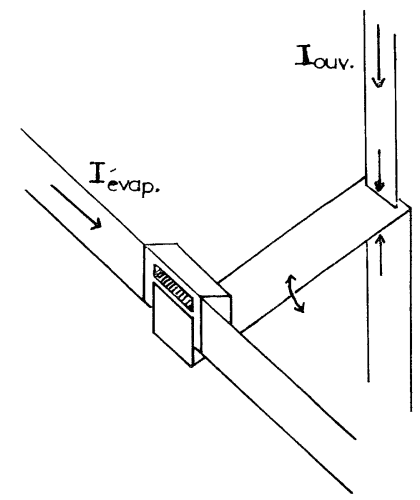

FIG. 3. - Creuset à ouverture. 
Grâce à ce procédé, nous avons pu réaliser des dépôts avec des vitesses de condensation relativement importantes sans que la pression résiduelle subisse de grande variation.

6. Accessoires de mesure. - La vitesse d'évaporation est maintenue constante par un asservissement utilisant comme capteur une jauge à ionisation [2] et permet de déterminer l'épaisseur des couches, après étalonnage, à l'extérieur de l'enceinte, par la méthode interférométrique de Nomarsky [3].

Un générateur à courant constant alimente la couche pendant la condensation, ce qui permet d'enregistrer la variation de sa résistance et de déterminer avec précision l'épaisseur critique à partir de laquelle on note une brusque diminution de la résistivité [4] (ceci nécessite, bien entendu, de réaliser le dépôt des électrodes (position II) avant celui de la couche mince (position I)).

Enfin, les pressions partielles, et en particulier celle d'oxygène, sont contrôlées pendant toute la durée de l'évaporation grâce à un analyseur de gaz résiduels du type Monopole.

\section{BIBLIOGRAPHIE}

[1] Salardenne (J.), Thèse de Doctorat d'État, Bordeaux, juin 1965.

[2] Dulhoste (J. C.), Thèse de $3^{\mathrm{e}}$ cycle, Bordeaux, juin 1968.
[3] Nomarski (G.) et Weir., (A. R.), Rev. Metallurg., 1955, 52, 2, 121 à 134.

[4] Komnik (Y. F.) et Palatnik (I. S.), Sov. Phys. Solid State, 1965, 7, 2, 429-431. 